Check for updates

Cite this: RSC Adv., 2017, 7, 41204

Received 19th June 2017

Accepted 15th August 2017

DOI: 10.1039/c7ra06828j

rsc.li/rsc-advances

\section{Design and synthesis of bi-functional Co- containing zeolite ETS-10 catalyst with high activity in the oxidative coupling of alkenes with aldehydes for preparing $\alpha, \beta$-epoxy ketones $\dagger$}

\author{
Jun Hu, ${ }^{a}$ Feifei Xia, ${ }^{a}$ Fengli Yang, ${ }^{a}$ Jushi Weng, ${ }^{a}$ Pengfei Yao, ${ }^{a}$ Chunzhi Zheng, ${ }^{a}$ \\ Chaojie Zhu, ${ }^{\text {b }}$ Tiandi Tang ${ }^{\mathrm{b}}$ and Wenqian Fu (D) *b
}

\begin{abstract}
Developing highly efficient heterogeneous catalysts for organic synthesis is of great importance in modern synthetic chemistry. In this work, Co (or Ni)-containing mesoporous zeolite ETS-10 (Co-METS-10 and NiMETS-10) with both metal and strong basic sites were synthesized and applied for the direct oxidative coupling of alkenes with benzaldehydes to synthesize $\alpha, \beta$-epoxy ketones. Co (or Ni)-METS-10 catalysts show high activity and product selectivity, as compared to metal-free mesoporous zeolite ETS-10 (METS-10). This feature is attributed to the fact that the highly dispersed Co (or Ni) species could facilitate the tert-butyl hydroperoxide transformation into more alkyloxy and alkylperoxy radicals, which triggers the alkenes undergoing radical addition with aldehyde and alkylperoxy to form $\beta$-peroxy ketones. Meanwhile, the basic sites on Co (or Ni)-METS-10 catalysts benefit the formation of $\alpha, \beta$-epoxy ketone from $\beta$-peroxy ketone.
\end{abstract}

\section{Introduction}

$\alpha, \beta$-Epoxy ketones are highly important and valuable compounds that have received much attention because of their wide application as versatile intermediates for transformations to many natural products and pharmaceuticals. ${ }^{1,2}$ Generally, these compounds are synthesized through a two-step procedure: synthesis of $\alpha, \beta$-unsaturated ketones through ClaisenSchmidt condensation reaction and subsequent epoxidation of $\alpha, \beta$-unsaturated ketones with peroxide catalyzed by environmentally unfriendly organic base in a homogenous reaction system. ${ }^{3-5}$ Recently, although Li et al. synthesized $\alpha, \beta$-epoxy ketones through iron-catalyzed carbonylation-peroxidation of alkenes to furnish $\beta$-peroxy ketones that were subsequently transformed into the corresponding epoxides in the presence of organic base catalyst, this approach is still employed in the twostep procedure. ${ }^{6}$ Furthermore, these reaction routes present the following disadvantages, such as the difficulty in separation of products and organic base from reaction systems and in reusing the catalysts, ${ }^{7,8}$ which severely limits their potential applications in industry. From a practical point of view, developing highly active heterogeneous catalysts with functionality is great

${ }^{a}$ School of Chemical and Environmental Engineering, Jiangsu University of Technology, Changzhou, Jiangsu 213001, PR China

${ }^{b}$ School of Petrochemical Engineering, Changzhou University, Changzhou, Jiangsu 213164, PR China. E-mail: fuwenqian@cczu.edu.com; Tel: +86-519-86330253

$\dagger$ Electronic supplementary information (ESI) available. See DOI: $10.1039 / \mathrm{c} 7 \mathrm{ra06828j}$ importance for synthesis of $\alpha, \beta$-epoxy ketones in green and sustainable route.

It has been reported that the metal site can facilitate tertbutyl hydroperoxide transformation to alkyloxy radicals, which can abstract hydrogen from aldehyde to give acyl radical., Subsequently, alkenes undergoing radical addition with acyl radical and hydroperoxides deliver $\beta$-peroxy ketones that can be transformed to corresponding epoxides catalyzed by organic base. ${ }^{\mathbf{1 0 - 1 2}}$ Thus, if the heterogeneous catalyst could contain both metal sites and basic sites, it would be realizable synthesis of $\alpha, \beta$-epoxy ketones through one-pot process. As well known, crystalline titanosilicate zeolite ETS-10 is porous materials with 12-membered ring network, and possesses strong basicity, ${ }^{\mathbf{1 3 , 1 4}}$ which can be as a solid basic catalyst to catalyze many reactions, such as knoevenagel reaction, ${ }^{15}$ aldol condensation ${ }^{16}$ and aryl amines oxidation reaction. ${ }^{\mathbf{1 7}}$ Therefore, it would be achievable that introducing metal species into zeolite ETS-10 to prepare bifunctional catalyst that has radical generation ability and base catalysis property for synthesis of $\alpha, \beta$-epoxy ketone compounds with high efficiency through one-step procedure.

Herein, as a continuous effort to developing novel zeolite catalysts for the organics transformations, ${ }^{\mathbf{1 8 - 2 1}}$ we report a facile methodology to synthesize Co-containing mesoporous zeolite ETS-10 (Co-METS-10) catalyst that shows very high activity in the formation of $\alpha, \beta$-epoxy ketones by oxidative coupling of alkenes with aldehydes through radical mechanism. This feature should be attributed to that the highly dispersed Co species on zeolite catalyst could facilitate the formation of more 
alkyloxy radicals, which prompt the alkenes underwent radical addition with aldehyde and alkylperoxy to form $\beta$-peroxy ketone intermediates, and the basic sites benefit the transformation from $\beta$-peroxy ketone to $\alpha, \beta$-epoxy ketone.

\section{Experimental}

\subsection{Material synthesis}

Co-containing mesoporous zeolite ETS-10 (Co-METS-10) was synthesized hydrothermally from a titanosilicate gel with a molar composition $3.7 \mathrm{Na}_{2} \mathrm{O} / 1.3 \mathrm{~K}_{2} \mathrm{O} / 1.0 \mathrm{TiO}_{2} / 6.7 \mathrm{SiO}_{2} /$ $0.6 \mathrm{TPOAB} / 0.18 \mathrm{CoO} / 163 \mathrm{H}_{2} \mathrm{O}$. TPOAB is $N, N$-dimethyl- $N$-octadecyl- $N$-(3-triethoxysilylpropyl)ammonium bromide as mesoscale template. In a typical run, $17.8 \mathrm{~mL}$ water glass was added in a $100 \mathrm{~mL}$ beaker. Then $10.0 \mathrm{~mL} \mathrm{NaOH}$ aqueous solution (5.38 $\mathrm{M})$ and $9.4 \mathrm{~mL} \mathrm{KOH}$ aqueous solution $(4.75 \mathrm{M})$ are mixed with the above solution in beaker under vigorous stirring until cooling to room temperature. After that, $13.1 \mathrm{~g} \mathrm{TiCl}$ solution ( $17 \mathrm{wt} \%$ in $\mathrm{HCl}$ ) was added into the beaker slowly followed by stirring for $240 \mathrm{~min}$. Finally, $3.5 \mathrm{~mL}$ TPOAB and $3 \mathrm{~mL}$ cobalt nitrate aqueous solution $(1.1 \mathrm{M})$ was added and further stirring for $2 \mathrm{~h}$. The obtained gel was transferred into Teflon-coated stainless-steel autoclave for crystallization at $230{ }^{\circ} \mathrm{C}$ for $72 \mathrm{~h}$. The resulting product was filtered, washed, and dried at $100^{\circ} \mathrm{C}$ overnight and calcined in air at $450{ }^{\circ} \mathrm{C}$ for $5 \mathrm{~h}$. Ni-containing mesoporous zeolite ETS-10 (Ni-METS-10) was synthesized by the same procedure except using nickel nitrate replacement of cobalt nitrate. For comparison, metal-free mesoporous ETS-10 (METS-10) and microporous ETS-10 zeolites were also synthesized by the same procedure in the absence of metal salts and template, respectively.

\subsection{Characterization}

X-ray diffraction (XRD) patterns of the samples were collected in a RIGAKU UltimalV diffractometer with $\mathrm{Cu} \mathrm{K} \alpha$ radiation $(\lambda=$ $1.548 \AA$ ̊). Nitrogen adsorption-desorption isotherms were measured at liquid nitrogen $\left(-196{ }^{\circ} \mathrm{C}\right)$ temperature using a Micromeritics ASAP 2020M automated apparatus. The sample was degassed for $8 \mathrm{~h}$ at $350{ }^{\circ} \mathrm{C}$ prior to the measurement. Surface area was calculated according to the BET equation. Mesopore volume was calculated by $t$-plot method. The content of the $\mathrm{Ni}$ (or $\mathrm{Co}$ ) in zeolite was analyzed by inductively coupled plasma optical emission spectroscopy (ICP-OES) with a PerkinElmer 2100DV emission spectrometer.

The morphology of the sample was observed with a field emission scanning electron microscope (SEM) on a SUPRA55 apparatus operated at an accelerating voltage of $5 \mathrm{kV}$. Transmission electron microscopy (TEM) experiment was performed on a JEM-2100F microscope with a limited line resolution capacity of $1.4 \AA$, under a voltage of $200 \mathrm{kV}$. Before characterization, the sample was cut into thin slices and dropped onto a $\mathrm{Cu}$-grid coated with carbon membrane. Ultraviolet-Visible (UV-Vis) spectrum was obtained on a Perkin-Elmer Lambda 25 spectrometer. X-ray photoelectron spectroscopic (XPS) measurement of the catalyst was performed on an ESCALAB MK II system.

\subsection{Activity test}

The oxidative coupling reactions of alkenes with aldehydes in the liquid-phase were conducted in glass reactor. In a typical run, $40 \mathrm{mg}$ catalyst, styrenes $(0.5 \mathrm{mmol})$, benzaldehydes $(2$ equiv., $1 \mathrm{mmol}$ ), $2 \mathrm{~mL}$ acetonitrile and tert-butyl hydroperoxide (TBHP, $1.0 \mathrm{mmol}, 70 \%$ aqueous solution) were injected into the glass reactor in sequence. The reaction mixture was then heated to desired temperature for continuous reaction for desired time. After the reaction was finished, the catalyst was separated by centrifugation to obtain the liquid phase for further analysis by an Agilent 7890B gas chromatograph equipped with a flame ionization detector.

\section{Results and discussion}

\subsection{Characterization}

The XRD patterns of the ETS-10, METS-10 and metalcontaining METS-10 samples in Fig. 1a give well-resolved diffraction peaks in the range $5-45^{\circ}$ with the ETS- 10 zeolite structure. ${ }^{14}$ Notably, Co and $\mathrm{Ni}$ crystalline phase are not detected in XRD pattern for Co-METS-10 and Ni-METS-10 samples, indicating that $\mathrm{Ni}$ and Co species are highly dispersed in the zeolites. Nitrogen adsorption-desorption isotherms of METS-10 and metal-containing METS-10 samples
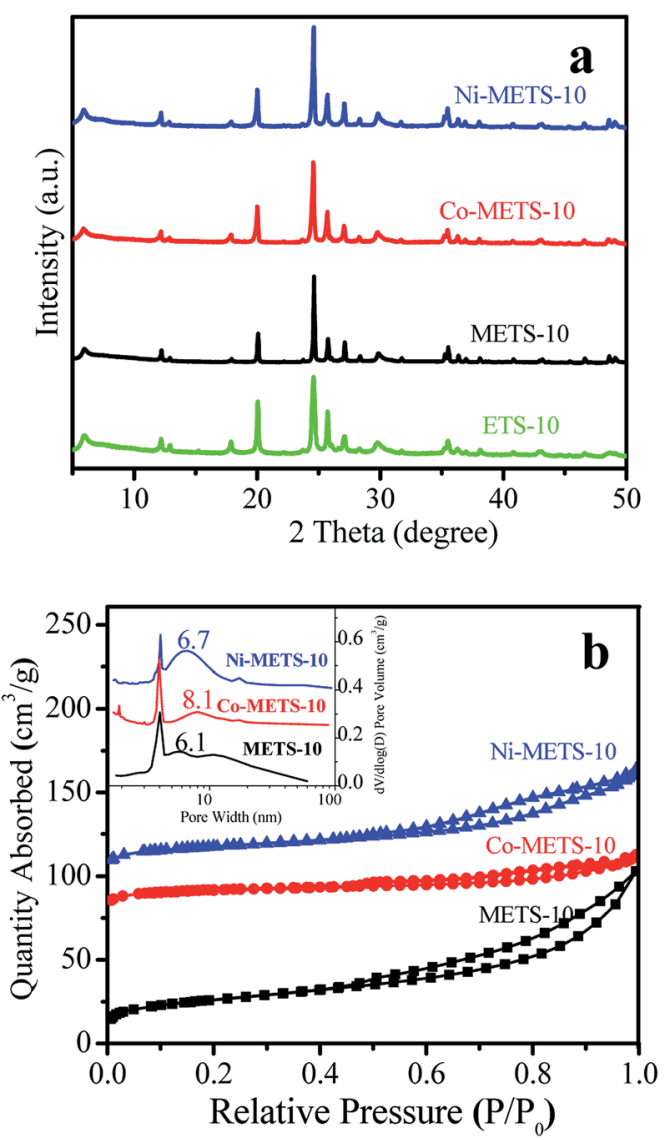

Fig. 1 (a) XRD patterns and (b) nitrogen adsorption-desorption isotherms and pore size distributions of the zeolite samples. 
Table 1 Texture parameters of the zeolite samples

\begin{tabular}{llllll}
\hline Samples & $\begin{array}{l}S_{\mathrm{BET}^{a}}{ }^{a} \\
\left(\mathrm{~m}^{2} \mathrm{~g}^{-1}\right)\end{array}$ & $\begin{array}{l}S_{\text {ext }}{ }^{b} \\
\left(\mathrm{~m}^{2} \mathrm{~g}^{-1}\right)\end{array}$ & $\begin{array}{l}V_{\text {micro }}{ }^{c} \\
\left(\mathrm{~cm}^{3} \mathrm{~g}^{-1}\right)\end{array}$ & $\begin{array}{l}V_{\text {meso }}{ }^{d} \\
\left(\mathrm{~cm}^{3} \mathrm{~g}^{-1}\right)\end{array}$ & $\begin{array}{l}\text { Metal } \\
\text { loading }^{e}(\mathrm{wt} \%)\end{array}$ \\
\hline ETS-10 & 335 & 16 & 0.12 & 0.03 & - \\
METS-10 & 330 & 65 & 0.11 & 0.13 & - \\
Ni-METS-10 & 320 & 75 & 0.11 & 0.12 & 1.8 \\
Co-METS-10 & 324 & 85 & 0.11 & 0.11 & 1.7
\end{tabular}

${ }^{a}$ BET surface area. ${ }^{b}$ External surface area (mesoporous surface area is included). ${ }^{c}$ Microporous volume. ${ }^{d}$ Mesoporous volume. ${ }^{e}$ The metal content was analyzed by ICP-OES.

give distinct hysteresis loop at relative pressure of $0.5-0.9$, suggesting the presence of mesoporous structure in the samples (Fig. 1b). ${ }^{21}$ The mesoporous sizes was centered at 6.1, 8.1 and $6.7 \mathrm{~nm}$ for METS-10, Co-METS-10 and Ni-METS-10 samples, respectively. Sample textual parameters presented in Table 1 show that Co-METS-10 and Ni-METS-10 have high mesoporous surface area ( 85 and $75 \mathrm{~m}^{2} \mathrm{~g}^{-1}$ ) and mesoporous volume $\left(0.11\right.$ and $\left.0.12 \mathrm{~cm}^{3} \mathrm{~g}^{-1}\right)$, which benefit the reactant diffusion, as compared to ETS-10 zeolite.

The SEM images display that the Co-METS-10 and Ni-METS10 catalysts have similar morphologies with particle size of 2-3 $\mu \mathrm{m}$ (Fig. $2 \mathrm{a}$ and $3 \mathrm{a}$ ). It seems that these particles are aggregated by nanosheets. TEM image of Co-METS-10 shows that the mesoporous (marked with white lines) are present in zeolite crystals, and very small Co clusters (black dots, marked with red

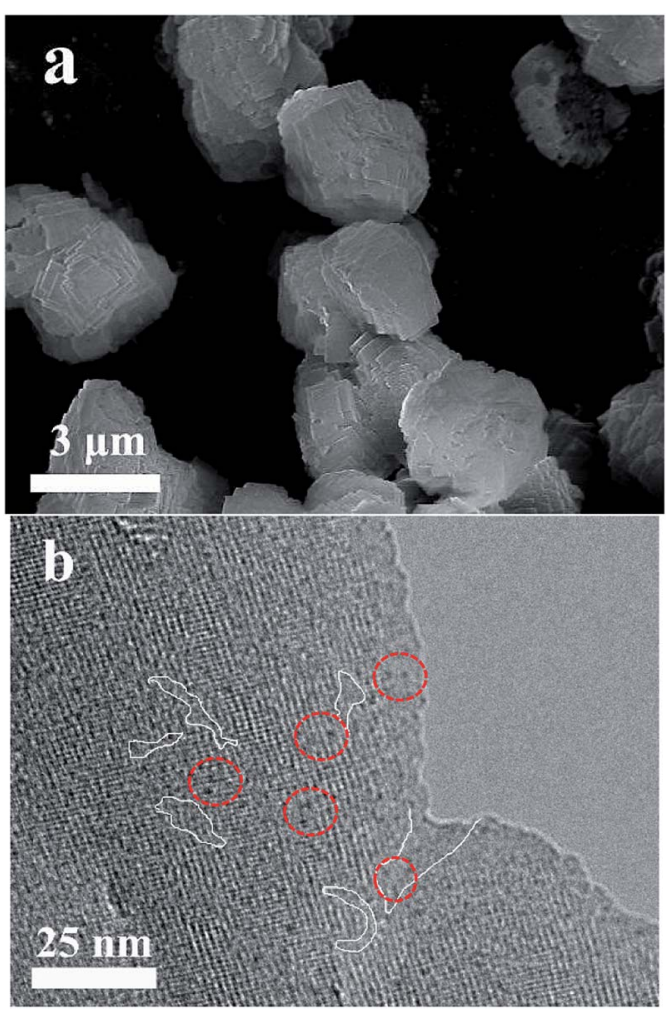

Fig. 2 (a) SEM and (b) TEM images of the Co-METS-10 zeolite. line) smaller than $1 \mathrm{~nm}$ are highly dispersed in microspores and mesopores (Fig. 2b). The mesopores (bright zones) are also observed in the TEM image of Ni-METS-10, and small $\mathrm{Ni}$ nanowires (black wire, about $1 \mathrm{~nm}$ diameter) with length of 5$10 \mathrm{~nm}$ are irregular dispersed in the micro-mesopores (Fig. 3b and $\mathrm{S} 1 \dagger)$.

The UV-Vis spectra of the METS-10, Co-METS-10 and NiMETS-10 samples are shown in Fig. 4. The absorption band at $280 \mathrm{~nm}$ can be assigned to the charge transfer transition involving $\mathrm{Ti}$ atoms in octahedral coordination in zeolite framework, which are in line with the reported literature. ${ }^{22}$ The electronic state of Co and Ni species are also investigated by XPS technique (Fig. 5). The Co 2p spectrum of Co-METS-10 sample has a main binding energy at $781.5 \mathrm{eV}$ with a shakeup feature at a higher binding energy, which is consistent with the characteristic of $\mathrm{Co}^{2+}$ species. $^{23}$ The Ni $2 \mathrm{p}$ spectrum of Ni-METS-10 shows a binding energy at $856.6 \mathrm{eV}$ associated with $\mathrm{Ni}^{2+}$ species, along with a shake-up peak at approximately $6.0 \mathrm{eV}$ higher than that of the $\mathrm{Ni}^{2+}$ species. ${ }^{24}$ These results indicate the presence of $\mathrm{Co}^{2+}$ and $\mathrm{Ni}^{2+}$ in the Co-METS-10 and Ni-METS-10 sample, respectively.

\subsection{Catalytic performance}

In order to evaluate the catalyst activity, the direct coupling of alkenes with aldehydes to synthesize $\alpha, \beta$-epoxy ketones was performed by choosing styrene and benzaldehyde as substrates at different temperatures. The results are shown in Fig. 6. At all

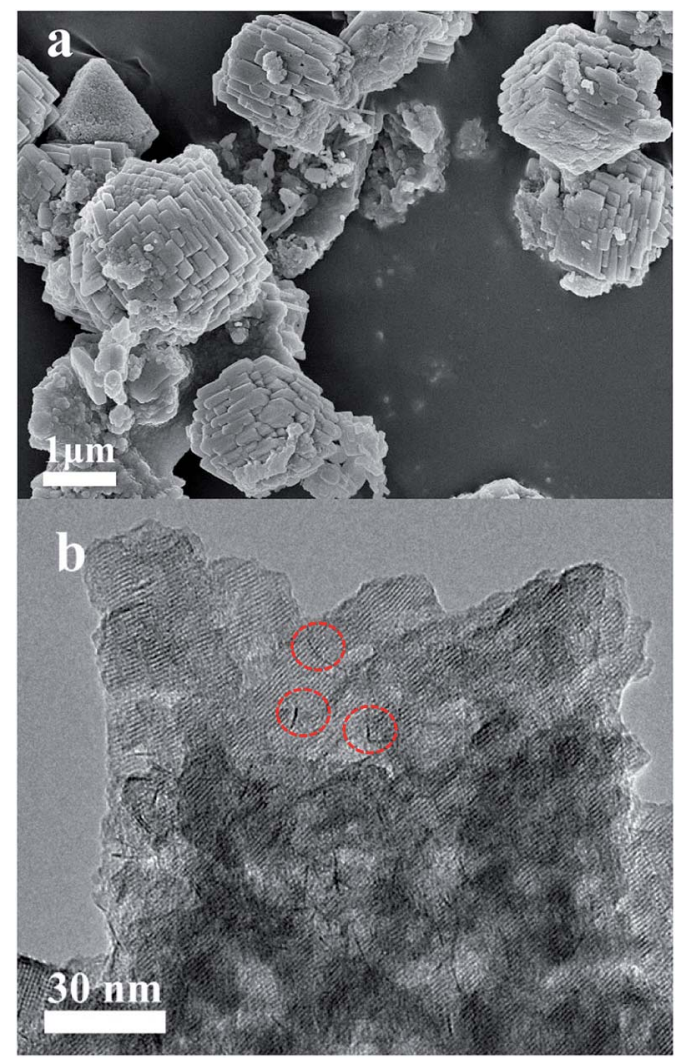

Fig. 3 (a) SEM and (b) TEM images of the Ni-METS-10 zeolite. 


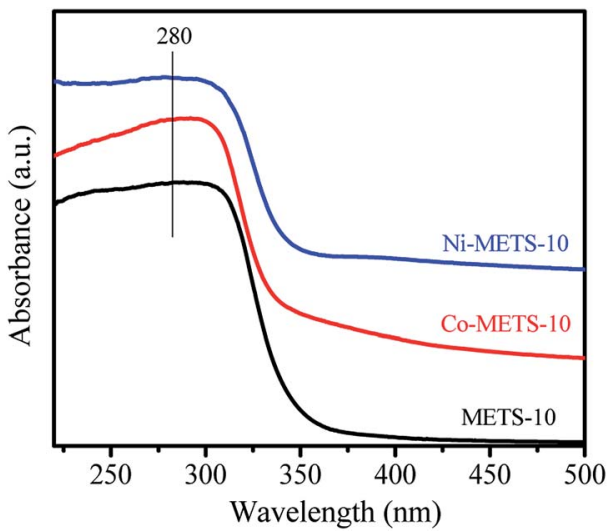

Fig. 4 UV-vis spectra of the zeolite samples.

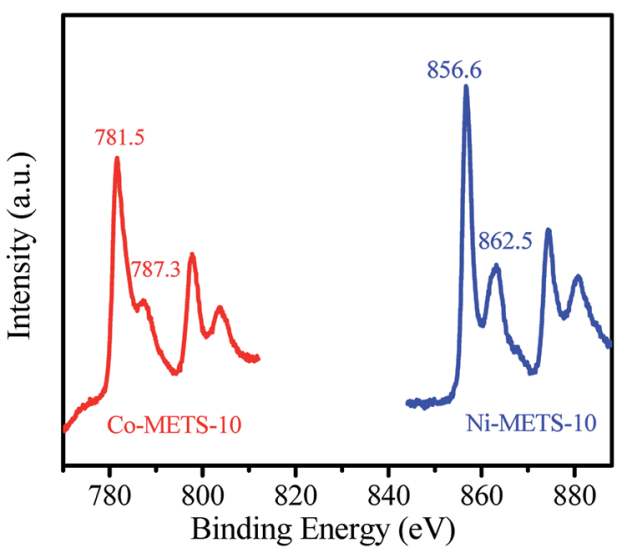

Fig. 5 XPS for Co $2 p$ and Ni $2 p$ of the zeolite samples.

reaction temperature, ETS-10 catalyst shows the lowest styrene conversion. This could be attributed to the mesoporous presence in METS-10, which could benefit the mass transfer. For the metal-containing METS-10 catalysts, Co-METS-10 (30\%) and NiMETS-10 (23\%) has poor styrene conversion at reaction temperature of $60{ }^{\circ} \mathrm{C}$, and are lower than metal-free METS-10 $(37 \%)$. Nevertheless, when the reaction was carried out at $70^{\circ} \mathrm{C}$, the styrene conversion over Co-METS-10 and Ni-METS- 10 was obviously improved ( $56 \%$ and $64 \%$ ), while that over METS10 is only $46 \%$. This result indicates that Co-METS-10 and NiMETS-10 have high reaction activity at relatively high temperature of $70{ }^{\circ} \mathrm{C}$. When the reaction was performed at $80^{\circ} \mathrm{C}$, the styrene conversions on Co-METS-10 and Ni-METS-10 are further improved, reaching $84 \%$ and $95 \%$, respectively.

To further investigate the catalytic performance, the target product selectivity of different zeolite catalysts was also compared. Fig. 7 shows styrene conversion and product selectivity with different reaction time at reaction temperature of $80{ }^{\circ} \mathrm{C}$. At the same reaction time, although Ni-METS-10 shows much higher styrene conversion than Co-METS-10, the target product selectivity over Ni-METS-10 is lower than that over CoMETS-10 catalyst. For example, at reaction time of $8 \mathrm{~h}$, styrene conversion over Co-METS-10 and Ni-METS-10 is 94 and 100\%,

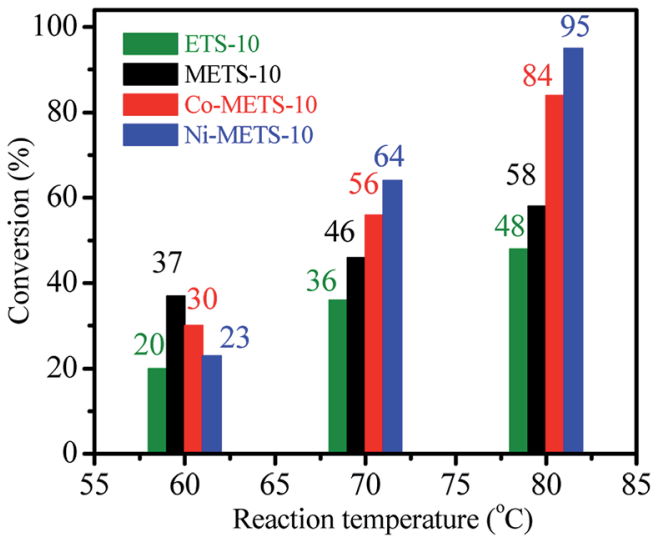

Fig. 6 Styrene conversions over Co-METS-10, Ni-METS-10, METS-10 and ETS-10 catalysts at different reaction temperature in the reaction time of $6 \mathrm{~h}$.

while the target product selectivity over Co-METS-10 and NiMETS-10 is 97 and $87 \%$. In addition, METS-10 shows the lowest styrene conversion and product selectivity at different reaction time among the three catalysts. Compared with METS-10, Co-METS-10 and Ni-METS-10 have equivalent mesoporous volume and external surface area (Table 1), but a higher activity and product selectivity are obtained on Co-METS-10 and Ni-METS-10 catalysts. These results indicate that $\mathrm{Co}(\mathrm{Ni})$-METS10 as bi-functional catalysts not only facilitate the enhanced reaction activity, but also benefit the formation of target product at relatively reaction temperature.

The superior catalytic performance of the Co(Ni)-METS-10 can be explained as follows. Generally, the oxidative coupling of alkenes with aldehydes over basic catalyst underwent radical reaction mechanism. ${ }^{6,12,14}$ The radical trapping experiment result also demonstrated that acyl radical was generated (the details please see ESI, Fig. S2 $\dagger$ ), suggesting that this oxidative coupling reaction over Co-METS-10 may perform through a radical mechanism. In this case, the generation of alkyloxy radical is crucial. Previous studies have reported that metal such as $\mathrm{Fe}^{2+}, \mathrm{Co}^{2+}$ and $\mathrm{Cu}^{2+}$ catalysts can promote the formation of alkyloxy radical from $t$-BuOOH by at relatively temperature $\left(80-120{ }^{\circ} \mathrm{C}\right){ }^{6,25,26}$ In our case, the metal $\mathrm{Co}^{2+}$ or $\mathrm{Ni}^{2+}$ species in METS-10 could facilitate the formation of the alkyloxy and alkylperoxy radicals at reaction temperature of $70-80^{\circ} \mathrm{C}$, which facilitate the formation of acyl radical that attack the styrenes to generate the $\beta$-peroxy ketones. In addition, our previous study reported that basic sites (marked as $\equiv \mathrm{TiO}_{6}{ }^{2-}$ ) on the METS-10 under heating not also help the $t$-BuOOH split into alkyloxy and alkylperoxy radicals, but also favor the transformation from $\beta$-peroxy ketones to $\alpha, \beta$-epoxy ketones. ${ }^{14}$ Thus, the formation of $\beta$-peroxy ketone intermediate was facilitated by the basic sites to transform $\alpha, \beta$-epoxy ketones over $\mathrm{Co}(\mathrm{Ni})$-METS-10 catalyst. As a conclusion, the presence of metal $\mathrm{Co}$ (or Ni) and basic sites on the $\mathrm{Co}(\mathrm{Ni})-\mathrm{METS}-10$ catalyst play a synergistic effect enhancement its catalytic activity at relatively high reaction temperature $\left(70-80{ }^{\circ} \mathrm{C}\right)$. The low activity of Co-METS- 10 catalyst at $60{ }^{\circ} \mathrm{C}$ could be attributed to that the density of basic sites on METS-10 

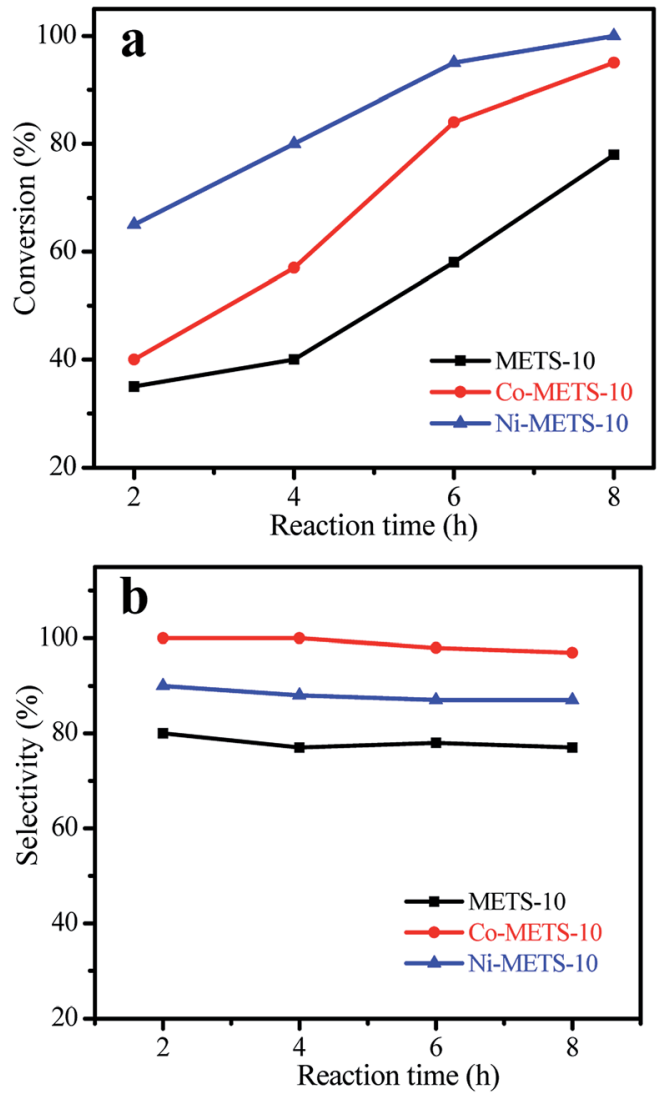

Fig. 7 (a) Styrene conversion and (b) product selectivity over CoMETS-10, Ni-METS-10 and METS-10 catalysts on different reaction time at reaction temperature of $80^{\circ} \mathrm{C}$.

is higher than on Co-METS-10 (Fig. S3, the details please see ESI $\dagger$ ).

On the basis of the above results and literature reports, ${ }^{6,14,25}$ a tentative reaction mechanism for Co-METS-10-catalyzed oxidative coupling reaction of styrene is given in Fig. 8 .
Alkyloxy and alkylperoxy radicals are generated over Co-METS10 catalyst (steps a and b). Subsequently, aldehydic hydrogen was abstracted from 2 by alkyloxy radical, giving acyl radical $\mathbf{4}$, which underwent radical addition with alkene giving the radical 5 and followed by radical coupling with alkylperoxy leading to the intermediate $\beta$-peroxy ketone 6. $\beta$-peroxy ketone 6 was catalyzed by basic sites $\left(\equiv \mathrm{TiO}_{6}{ }^{2-}\right)$ on Co-METS-10 to form the final product 3 .

Encouraged by the promising results, the scope of this oxidative coupling reaction over Co-METS-10 catalyst was investigated through choosing different aldehydes and styrenes, and the results are summarized in Table 2. Co-METS10 catalyst tolerates various benzaldehydes with electrondonating (methoxy, methyl) and electron-draw (chlorine, bromine) substituents, giving high activity and target product selectivity (3a-3e). Meanwhile, styrenes with halogen

Table 2 The oxidative coupling of styrenes with benzaldehydes at $80^{\circ} \mathrm{C}$ for $8 \mathrm{~h}^{a}$

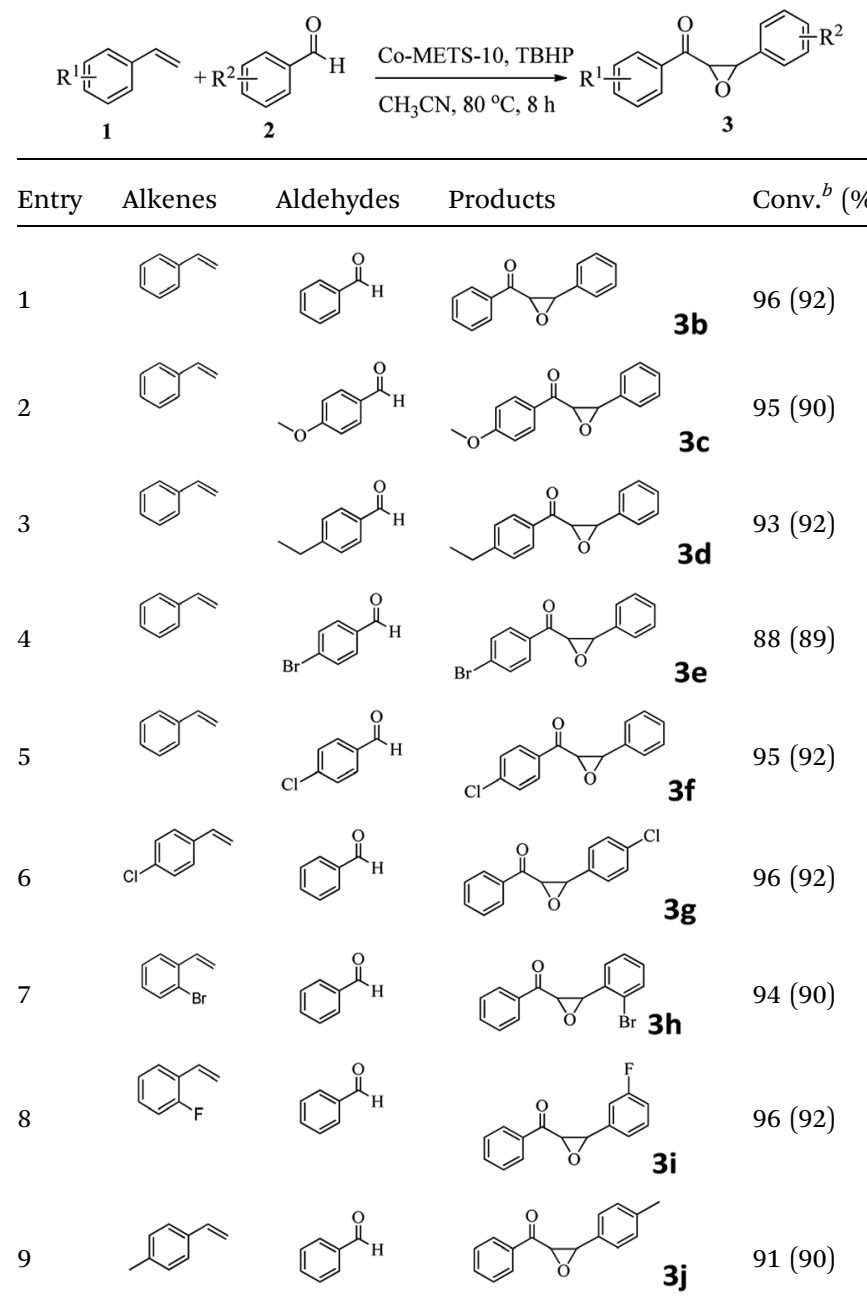

${ }^{a}$ Conditions: styrenes $(0.5 \mathrm{mmol})$, benzaldehydes $(1.0 \mathrm{mmol})$, TBHP $(1.0 \mathrm{mmol})$, Co-METS-10 (40 mg), MeCN (2.0 mL), $80{ }^{\circ} \mathrm{C}, 8 \mathrm{~h}$. ${ }^{b}$ Obtained by GC analysis, the data out of parenthesis is conversion, and in parenthesis is selectivity.

Fig. 8 Proposed reaction mechanism. 
substituents at the ortho, meta and para positions are also suitable for this transformation and give the products in good yields (3f-3h). In addition, the reagent 4-methyl styrene is also successfully applied to this transformation, affording the desired product in gratifying yield (3i).

\section{Conclusions}

In summary, the Co (or Ni)-containing mesoporous zeolite ETS10 was directly synthesized by templating with a mesoscale silane surfactant through hydrothermal method. Co-METS-10 and Ni-METS-10 catalysts show high activity in the oxidative coupling reaction of styrenes with benzaldehydes through onepot synthetic procedure. In addition, Co-METS-10 exhibits the highest product selectivity to form $\alpha, \beta$-epoxy ketones. Co (or Ni)METS-10 has bi-functional characteristic with transition-metal and strong basicity sites. The highly dispersed Co and $\mathrm{Ni}$ species facilitate the generation of the alkyloxy and alkylperoxy radicals from $t$-BuOOH, leading to the occurrence of radical addition for the alkenes with aldehyde and alkylperoxy to form $\beta$-peroxy ketones. Meanwhile, the basic sites on Co (or Ni)METS-10 catalyst favor the transformation from intermediate $\beta$-peroxy ketone to $\alpha, \beta$-epoxy ketones, resulting in the enhanced catalytic activity.

\section{Conflicts of interest}

There are no conflicts to declare.

\section{Acknowledgements}

This work was supported by the National Natural Science Foundation of China (U1463203, 21476030 and U1662139).

\section{Notes and references}

1 D. E. Bergbreiter, J. H. Tia and C. Hongfa, Chem. Rev., 2009, 109, 530-582.

2 M. J. Climent, A. Corma and L. Sara, Chem. Rev., 2011, 111, 1072-1133.

3 B. M. Choudary, M. L. Kantam, K. V. S. Ranganath, K. Mahendar and B. Sreedhar, J. Am. Chem. Soc., 2004, 126, 3396-3397.

4 Y. Wang, J. Ye and X. Liang, Adv. Synth. Catal., 2007, 349, 1033-1036.
5 M. Marigo, J. Franzén, T. B. Poulsen, W. Zhuang and K. A. Jørgensen, J. Am. Chem. Soc., 2005, 127, 6964-6965.

6 W. Liu, Y. Li, K. Liu and Z. Li, J. Am. Chem. Soc., 2011, 133, 10756-10759.

7 M. J. Climent, A. Corma and S. Iborra, Chem. Rev., 2011, 111, 1072-1133.

8 À. Molnár, Chem. Rev., 2011, 111, 2251-2320.

9 J. Wang, C. Liu, J. Yuan and A. Lei, Angew. Chem., Int. Ed., 2013, 125, 2312-2315.

10 W.-T. Wei, X.-H. Yang, H.-B. Li and J.-H. Li, Adv. Synth. Catal., 2015, 357, 59-63.

11 Q. Ke, B. Zhang, B. Hu, Y. Jin and G. Lu, Chem. Commun., 2015, 51, 1012-1015.

12 V. Ashokkumar and A. Siva, Org. Biomol. Chem., 2017, 15, 2551-2561.

13 P. D. Southon and R. F. Howe, Chem. Mater., 2002, 14, 42094218.

14 M. Xiang, X. Ni, X. Yi, A. Zheng, W. Wang, M. He, J. Xiong and T. Tang, ChemCatChem, 2015, 7, 521-525.

15 X. Ni, M. Xiang, W. Fu, Y. Ma, P. Zhu, W. Wang, M. He, K. Yang, J. Xiong and T. Tang, J. Porous Mater., 2016, 23, 423-429.

16 A. Philippou and M. W. Anderson, J. Catal., 2000, 189, 395400.

17 S. B. Waghmode, S. M. Sabne and S. Sivasanker, Green Chem., 2001, 3, 285-288.

18 W. Fu, T. Liu, Z. Fang, M. Ma, X. Zheng, W. Wang, X. Ni, M. Hu and T. Tang, Chem. Commun., 2015, 51, 5890-5893.

19 W. Fu, Y. Feng, Z. Fang, S. Chen, T. Tang, Q. Yu and T. Tang, Chem. Commun., 2016, 52, 3115-3118.

20 T. Tang, L. Zhang, H. Dong, Z. Fang, W. Fu, Q. Yu and T. Tang, RSC Adv., 2017, 7, 7711-7717.

21 H. Dong, L. Zhang, Z. Fang, W. Fu, T. Tang, Y. Feng and T. Tang, RSC Adv., 2017, 7, 22008-22016.

22 S. B. Hong, S. J. Kim and Y. S. Uh, Korean J. Chem. Eng., 1996, 13, 419-421.

23 M. C. Biesinger, B. P. Payne, A. P. Grosvenor, L. W. M. Lau, A. R. Gerson and R. S. C. Smart, Appl. Surf. Sci., 2011, 257, 2717-2730.

24 W. Fu, L. Zhang, D. Wu, Q. Yu, T. Tang and T. Tang, Ind. Eng. Chem. Res., 2016, 55, 7085-7095.

25 J. Wang, C. Liu, J. Yuan and A. Lei, Angew. Chem., Int. Ed., 2013, 52, 2256-2259.

26 Y. R. Li, M. Wang, W. Fan, F. Qian, G. Li and H. Lu, J. Org. Chem., 2016, 81, 11743-11750. 